\title{
Comparison of Proposed Path Selection Protocols for IEEE 802.11s WLAN Mesh Networks
}

\author{
Sana Ghannay, Sonia Mettali Gammar and Farouk Kamoun
}

CRISTAL Lab, National School of Computer Sciences, ENSI, 2010, Manouba e-mail: \{sana.ghanlnay,sonia.gammar,frk.kamoun\}@ cristal.rnu.tn

\begin{abstract}
IEEE 802.11s wireless LAN mesh network technology is the next step in the evolution of wireless architecture. A WLAN mesh network consists of WLAN devices with relay functions that communicate directly. In this type of networks, path selection is based on two protocols: HWMP and RA-OLSR. This paper presents a detailed study of the performance of the proposed path selection algorithms for IEEE 802.11s WLAN mesh networks based on the current draft standard D1.08 from January 2008 under different scenarios to provide conditions of the applicability of the protocols.
\end{abstract}

\section{Introduction}

WLAN wireless mesh networks are anticipated to deliver wireless services for a large variety of applications in personal, local, campus, and metropolitan areas. The main characteristic of wireless mesh networking is the communication between nodes over multiple wireless hops on a network [1]. Indeed, WLAN mesh networks consist of Mesh Point and Mesh Stations, where mesh point have minimal mobility and form the backbone of WLAN mesh network. They provide network access for both mesh and conventional stations. Furthermore, the integration of WLAN mesh networks with other networks such as the Internet, cellular, etc., can be accomplished through the gateway and bridging functions in the Mesh Point [2].

The standardization of WLAN mesh network in IEEE 802.11s is work in progress. The goal of the IEEE 802.11s group is the development of a flexible and extensible standard for wireless mesh network based on IEEE 802.11 [1]. Many research challenges remain in wireless mesh networks such as mesh Medium Access Coordination, mesh security, mesh routing, etc.

One of the key functionalities of IEEE 802.11s is the wireless multi-hop routing, which sets up the paths for the wireless forwarding. For that reason, efficient routing protocols have to provide paths through the wireless mesh and react to dynamic changes in the topology so that mesh nodes can communicate with each other even if they are not in direct wireless range. IEEE 802.11s proposed two path selection protocols HWMP(Hybrid Wireless Mesh Protocol) and RA-OLSR ( Radio-Aware Optimized Link State Routing). In this paper, we present HWMP and RA-OLSR, we evaluate their performance and we compare them through simulation. 
The remainder of the paper is structured as follows. Section 2 gives a brief overview of IEEE 802.11s future standard. Path selection protocols HWMP and RA-OLSR are explained in section 3 . Section 4 presents simulation results and compares the proposed path selection protocols.

\section{The IEEE 802.11s standard}

The IEEE 802.11s working group specifies an extension to the IEEE 802.11 MAC to solve the interoperability problem by defining an architecture and protocol that support both broadcast/multicast and unicast delivery using "radio-aware" metrics over self-configuring multi-hop topologies [1]. The IEEE 802.11s WLAN mesh network architecture is composed of (Fig. 1) [3]:

- MP (Mesh Point): is a dedicated node for forwarding packets on behalf of other MP that may not be within direct wireless transmission range of their destinations

- Mesh Access Point (MAP): is a particular MP that provides the network access to the clients or stations

- MPP (Mesh Portal Point): is a particular MP that acts as a bridge to access external networks like Internet and WiMax

- STA (Station): is connected via a MAP to the mesh network

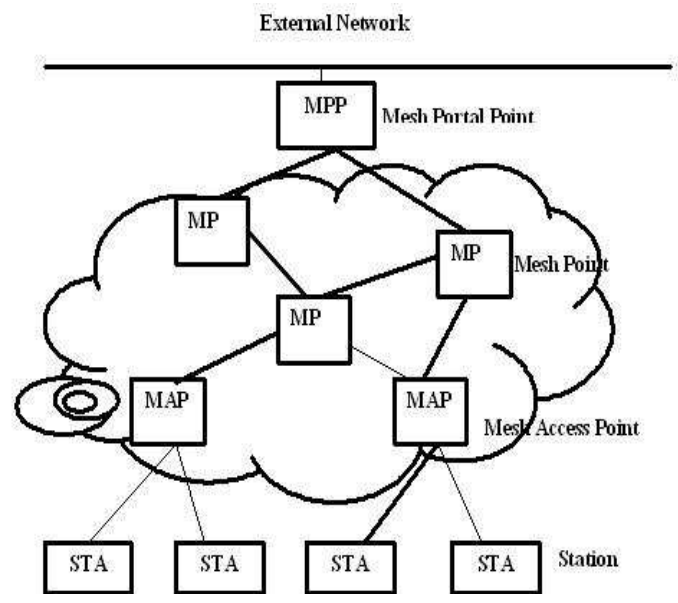

Fig. 1 IEEE 802.11s WLAN Mesh network architecture 


\section{Path selection protocols}

Each network uses a single method to determine paths through the Mesh. The standard includes a default mandatory path selection protocol HWMP to ensure interoperability and an optional routing protocol called RA-OLSR. Moreover, the proposed IEEE 802.11s define a default radio-aware path selection metric called the Airtime Link Metric (ALM). The ALM metric is calculated as [1] :

$$
C_{a}=\left[O+\frac{B_{t}}{r}\right] \frac{1}{1-e_{f}}
$$

$O$ and $B_{t}$ are constant. $r$ is the transmission bit rate. $e_{f}$ is the frame error rate for the Test Frame of size $B_{t}$. ALM estimates the quality of a link by taking into account the packet loss probability as well as the bit rate of the link.

\subsection{Hybrid Wireless Mesh Protocol}

As a hybrid protocol, HWMP combines the flexibility of on-demand routing with proactive topology tree extension [1]. HWMP contains two operating modes : a reactive mode and a proactive mode. As a hybrid protocol, HWMP combines the flexibility of on-demand routing with proactive topology tree extension [1]. The reactive mode defines RM-AODV which is an extension of AODV using ALM. If the proactive mode is selected, a Mesh Portal Point (MPP) is configured as Root and HWMP sets up a tree to this Root. This second mode of operation of HWMP called the Proactive tree building mode can be performed with Proactive PREQ (Proactive Path REQuest) or Proactive RANN (Proactive Root ANNouncement) depending on the configuration of the root [4].

\subsubsection{Reactive routing mode in HWMP}

RM-AODV is an adaptation of AODV [5]. In RM-AODV, if a source MP needs a route to a destination, it broadcasts a PREQ (Path REQuest) with the destination MP address specified in the destination field and the ALM metric is initialized to 0. When an MP receives a PREQ, it creates a path to the source or updates its current path if the PREQ contains a greater sequence number or the sequence number is the same as the current path and the PREQ offers a better metric than the current path. Whenever an MP forwards a PREQ, the metric field in the PREQ is updated to reflect the cumulative metric of the path to the PREQ's source. After creating or updating a path to the source, the destination MP sends a unicast PREP back to the source.

Intermediate MPs create a path to the destination on receiving the PREP and also forward the PREP toward the source. When the source receives the PREP, it creates a path to the destination. If the destination receives further PREQs with a better metric then the destination updates its path to the source to the new path and also 
sends a PREP to the source along the updated path. A bidirectional, best metric endto-end path is established between the source and destination. As long as the route remains active, it will continue to be maintained. A route is considered active as long as there are data packets periodically travelling from the source to the destination along that path. Once the source stops sending data packets, the links will time out and eventually be deleted from the intermediate node routing tables. If a link break occurs while the route is active, the node upstream of the break propagates a route error PERR (Path ERRor) message to the source node to inform it of the unreachable destinations. After receiving the PERR, if the source node still desires the route, it can reinitiate route discovery.

\subsubsection{Proactive Tree mode}

There are two mechanisms for proactively disseminating path selection information for reaching the Root MP : Proactive PREQ Mechanism and Proactive RANN Mechanism.

\section{Proactive PREQ Mechanism}

The PREQ tree building process begins with a proactive Path Request message broadcast by the Root MP. The PREQ contains the path metric set to 0 and a sequence number. The proactive PREQ is sent periodically by the Root MP, with increasing sequence numbers.

An MP hearing a proactive PREQ creates or updates its forwarding information to the Root MP, updates the metric and hops count of the PREQ, records the metric and hop count to the Root MP and then transmits the updated PREQ. Each MP may receive multiple copies of a proactive $\mathrm{PREQ}$, each traversing a unique path from the Root MP to the MP. An MP updates its current path to the Root MP if and only if the PREQ contains a greater sequence number or the sequence number is the same as the current path and the PREQ offers a better metric than the current path to the Root MP.

\section{Proactive RANN Mechanism}

The Root MP periodically propagates a RANN message into the network. The information contained in the RANN is used to disseminate path metrics to the Root MP. Upon reception of a RANN, each MP that has to create or refresh a path to the Root MP sends a unicast PREQ to the Root MP via the MP from which it received the RANN. The unicast PREQ follows the same processing rules defined in the on demand mode. The Root MP sends PREP in response to each PREQ. The unicast 
PREQ creates the reverse path from the Root MP to the originating MP while the PREP creates the forward path from the MP to the Root MP.

\subsection{Radio-Aware Optimized Link State Routing Protocol}

RA-OLSR protocol is a proactive link-state wireless mesh path selection protocol based on Optimized Link State Routing (OLSR) [6] protocol and the Fisheye State Routing (FSR) [7] protocol and uses a radio aware metric for forwarding path and multipoint relay set calculation.

OLSR is based on MPR flooding technique to reduce the number of transmission as compared to classical flooding mechanism where each node forward all received packets. In OLSR, a node selects a minimal subset of its one hop neighbors set to cover all its two hop neighbors set to act as multipoint relaying nodes. The process is based on information acquired from Hello messages which are containing list of it neighbors' links. When a node sends/forwards a broadcast Topology Control (TC) message, containing the topology information necessary to build the routing tables, only its MPR nodes forward the message reducing duplicate retransmission. In order to reduce the routing overhead (TC message) of OLSR, RA-OLSR adopts FSR technique.

FSR [7] is a proactive routing protocol based on link state routing. In FSR, information about closer nodes is exchanged more frequently than it is done about further nodes. So each node has the most up to date information about all nearby nodes and the accuracy of information decreases as the distance from node increases.

In RA-OLSR, there are different frequencies for propagating the TC message to different scopes so that the fisheye scope technique allows exchanging link state message at different intervals for nodes within different fisheye scope distance, leading to a reduction of the link state message size.

\section{Comparison}

In this section, we compare mesh typical routing protocols according to different criterias. (Table. 1) resumes the characteristics of each routing protocols. Route Computation indicates when the route is computed. There are two cases: reactive and proactive. Stored Information denotes the information stored in each node. As for Update Period, it is mainly applicable to proactive protocols and assumes values. The Update information is the message used to update routing table entry concerning an Update destination. 
Table 1 Comparison of Typical Routing Protocols

\begin{tabular}{lllll}
\hline Characteristic & RM-AODV & Proactive PREQ & Proactive RANN & RA-OLSR \\
\hline Route computation & Reactive & Proactive & Proactive & Proactive \\
structure & Flat & Tree & Tree & Flat \\
Stored information & Next hop for destination & Next hop for Root Next hop for Root Entire topology \\
Update period & Even driven & Periodical & Periodical & Periodical \\
Update information & PERR & PREQ & RANN & Hello and TC \\
Update destination & Source & Root & Root & All nodes \\
Method & Unicast & Tree Broadcast & Tree Broadcast & Broadcast \\
Routing metric & ALM & ALM & ALM & ALM \\
\hline
\end{tabular}

\section{Performance evaluation}

The purpose of our simulations is to uncover in which situations the individual protocols have their strengths and weaknesses, rather than to promote one protocol as generally better than the others. Thus, in order to avoid getting results which favor either of the protocols, we apply a strategy of specifying a set of parameters (number of nodes, number of CBR connections etc), from which a large number of scenarios are randomly generated. These scenarios will be different, yet have the same overall characteristics.

In our performance evaluation we have implemented a sub layer called IEEE 802.11s between IP and MAC layers. This sub layer contains two modules: Path selection module and Measurement module. The Path selection module contains functions for determining routes to transfer packets to their destination using MAC address as identifier. The Mesh network Measurement module contains functions for calculating and advertising radio metric used by routing protocols. It uses two frames: Test Frame and Link State Announcement frame which are mesh management frames. Test Frame has a fixed size and it is transmitted every Test Frame interval. Each MP computes the number of received Test Frame in a fixed period of exchange. It calculates the loss probability as the ratio between the number of received Test Frames and the number of sent Test Frames. Link State Announcement Frame is used to advertise periodically the loss probability to compute Frame Error Rate $e_{f}$ in both the forward and reverse directions to account for data as well as ACK.

In our implementation, we use the novel MAC frame format described by IEEE 802.11s future standard [1]. This MAC frame format is an extension of the existing data frame format. 


\subsection{Simulation parameters}

We conduct our simulations using the Glomosim simulator [8]. We base all our scenarios on the following basic parameters (Table. 2):

Table 2 Simulation parameters

\begin{tabular}{ll}
\hline Simulation time & $200 \mathrm{~s}$ \\
Network density & 1 over $80 \mathrm{~m}^{2}$ \\
Transmission range & $200 \mathrm{~m}$ \\
Bandwidth & $2 \mathrm{Mbit} / \mathrm{s}$ \\
Node placement & Uniform \\
Traffic type & $\mathrm{CBR}$ \\
Packet size & $512 \mathrm{bytes}$ \\
Packet rate & $10 \mathrm{pkts} / \mathrm{s}$ \\
RANN interval & $3 \mathrm{~s}$ \\
Proactive PREQ interval & $3 \mathrm{~s}$ \\
Test Frame interval & $1 \mathrm{~s}$ \\
Test Frame size & $8192 \mathrm{bits}$ \\
Test Frame period of exchange & $16 \mathrm{~s}$ \\
Link State Announcement Frame & $4 \mathrm{~s}$ \\
\hline
\end{tabular}

\subsection{Simulation results}

Simulations have been conducted with varying number of nodes and varying number of CBR connections to examine the protocols in different scenarios. Comparisons have been done on the following performance metrics: Packet delivery ratio, End to end delay and Routing overhead.

- Packet delivery ratio: the ratio between the number of packets delivered to the receiver and the number of packets sent by the source.

- End to end packet delay: the mean time in second taken by data packets to reach their respective destinations.

- Routing overhead: the number of routing bytes required by the routing protocol to construct and maintain its routes.

We have defined two scenarios. In the first scenario, the traffic is distributed over the network. Source and destination of any CBR connections are distributed among all nodes and are chosen randomly. In the second scenario, all the CBR connections are intended to Root. 

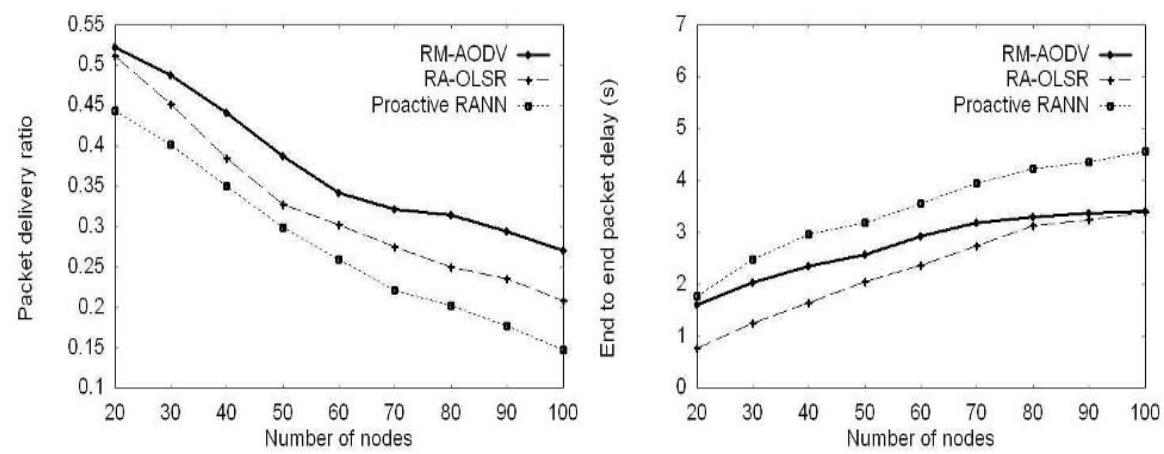

Fig. 2 Packet delivery ratio as a function of num- Fig. 3 End to end packet delay as a function of ber of nodes number of nodes
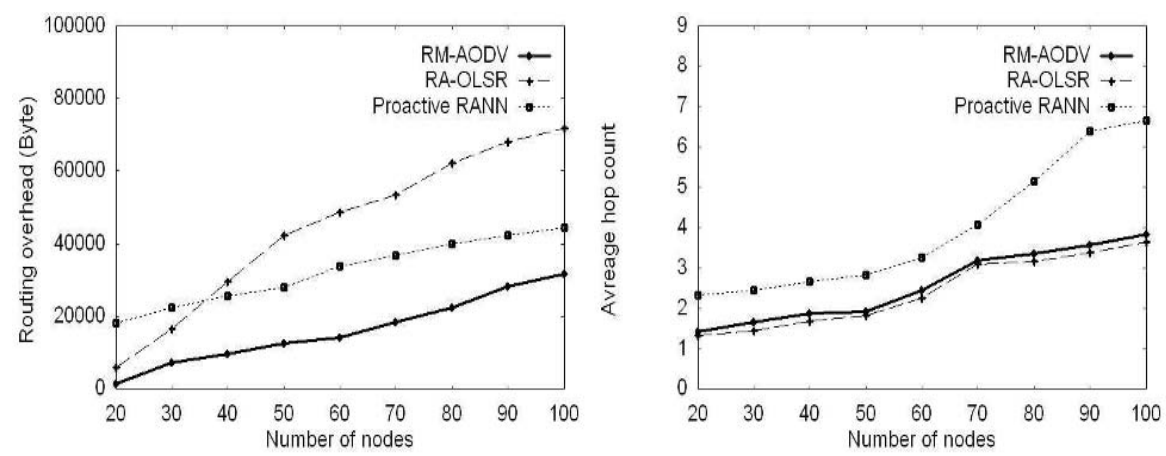

Fig. 4 Routing overhead as a function of number Fig. 5 Number of hops in the path as a function of nodes of number of nodes

\subsubsection{Distributed traffic}

In figures 2, 3 and 4, we report the performance in term of packet delivery ratio, end to end packet delay and routing overhead as a function of the number of nodes over RM-AODV, RA-OLSR, and Proactive RANN for 20 CBR connections. We noted that, as the number of nodes increases, the packet delivery ratio decreases while the end to end packet delay increases for all routing protocols. Indeed, as the routing overhead increases, so does the interference and contention.

Fig. 2 presents the packet delivery ratio against the number of nodes. RM-AODV outperforms RA-OLSR because it introduces less routing overhead. In RM-AODV, as the network is without mobility, traffic control is sent only at the beginning of any CBR connection. However, in RA-OLSR, traffic control is sent periodically and it increases as the network dimensions. Moreover, due to the use of the ALM metric instead of the number of hops, we noted that the average routes length for CBR connections established by RA-OLSR and RM-AODV are approximately equal (Fig. 5). In figure 3, we observe that RA-OLSR consistently presents the lowest delay for 

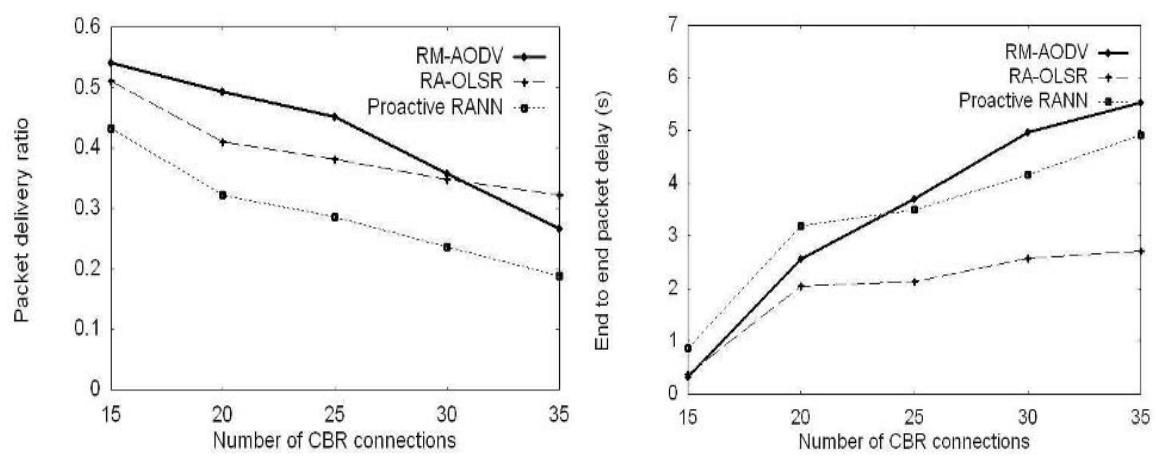

Fig. 6 Packet delivery ratio as a function of num- Fig. 7 End to end packet delay as a function of ber of CBR connections number of CBR connections
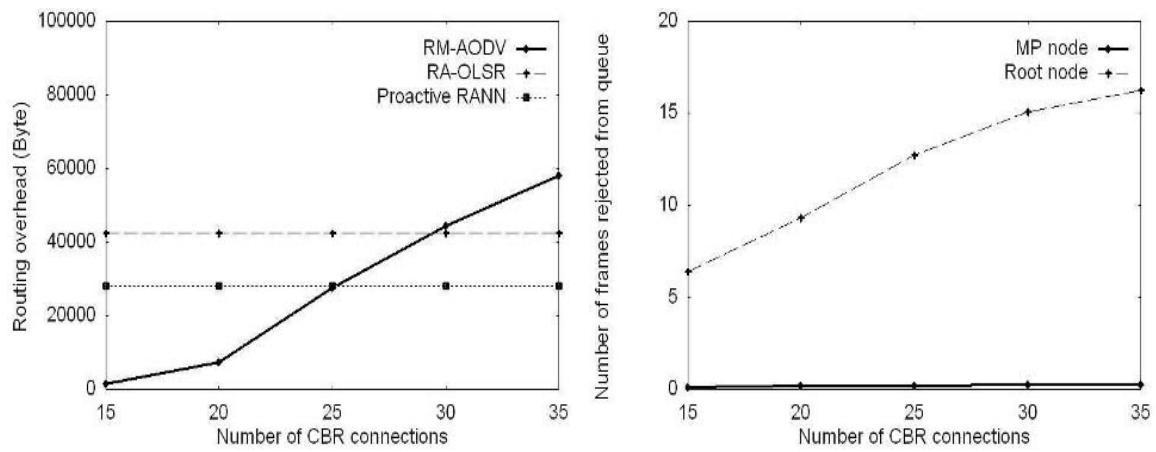

Fig. 8 Routing overhead as a function of number Fig. 9 Number of frame rejected from queue as of CBR connections a function of number of nodes

successful packets, regardless of number of nodes. This may be explained by the fact that RA-OLSR is a proactive protocol: when a packet arrives at a node, it can immediately be forwarded or dropped. In reactive protocols, if there is no route to a destination, packets to that destination will be stored in a buffer while a route discovery is conducted. This may cause longer delays. The difference between RA-OLSR and RM-AODV decreases regardless the number of nodes. From a certain threshold (80 nodes) both curves get closer. Indeed, in figure 4, we clearly see an important difference in the routing overhead generated by RA-OLSR and RM-AODV. For number of nodes below 40, the control traffic overhead of RA-OLSR is composed of Hello messages only and it gets closer to the routing overhead of RM-AODV. However, as the number of node increases, the broadcast of TC messages introduces a large control traffic overhead.

The Proactive RANN protocol has the worst performance regardless the number of nodes. In fact, all the CBR connections pass through the Root. Besides, Root sends and receives all control traffic. This node becomes overloaded and it rejects incoming packets. Moreover, Proactive RANN has the longest number of hops for CBR 
paths (Fig. 5) (Proactive RANN has approximately the double number of hops then RA-OLSR and RM-AODV). This increases both the overhead and the delay and decreases the packet delivery ratio.

We have investigated another set of simulations varying the number of CBR connections in a network of 50 nodes. For more than approximately 30 concurrent CBR connections, the packet delivery ratio of RA-OLSR is higher than that of RM-AODV (Fig. 7). In fact, with a large number of concurrent CBR connections, extra control traffic overhead is consumed by route establishment in the reactive protocols (as showed in (Fig. 8)). This leaves less available bandwidth for data traffic and increases probability of loss due to collisions.

Figure 7 shows that RA-OLSR has the lowest end to end packet delay. In fact, RAOLSR is an optimization over a classical link-state routing protocol, tailored for WLAN mesh networks, as such, it inherits all the benefits of link-state routing protocols, including the immediate availability of routes when needed, which greatly reduces the initial route setup delay.

Proactive RANN has the lowest packet delivery ratio and the highest end to end packet delay until $25 \mathrm{CBR}$ connections. Indeed, as the number of CBR connections increases, the load of Root increases leading to more rejected frames from Root queue as shown in (Fig. 9). It presents the average number of rejected frames from queue per second for the Root and an MP. We noted that the number of Root rejected frames is very high compared to an ordinary MP.

We have investigated another set of simulation where the traffic is as follows: half of data CBR connections are intended to Root and the rest is distributed over the network. We observed a similar behavior for the three algorithms.

\subsubsection{Traffic intended to Root}

The main purpose of mesh networks is to allow Internet access. By this way, most of traffic is from MP to Root. Therefore, we have defined another scenario where the most of CBR connections are sent to Root. In this scenario, performance of Proactive PREQ protocol is evaluated in addition to RA-OLSR, RM-AODV and Proactive RANN as a function of number of nodes and number of CBR connections. Indeed, Proactive PREQ provides only routes to Root.

Figures 10 and 11 depict the packet delivery ratio and the end to end packet delay respectively regardless the number of nodes. They show that all mode of HWMP outperforms RA-OLSR. Indeed, in RM-AODV, as all CBR connections are intended to Root, the probability to find an existing path established to the root increases leading to less delay and overhead and more delivered packet. As regards Proactive PREQ protocol, it introduces the least routing overhead. In fact, the intention of the Proactive PREQ mode is a "lightweight" HWMP topology formation where the routing overhead for the proactive extension is kept at a minimum. The broadcast PREQ messages set up a tree that contains paths from all mesh points to the Root, but mesh points are not registered proactively at the Root. Besides, we can see in (Fig. 11)that Proactive PREQ introduces less delay than RM-AODV due its 

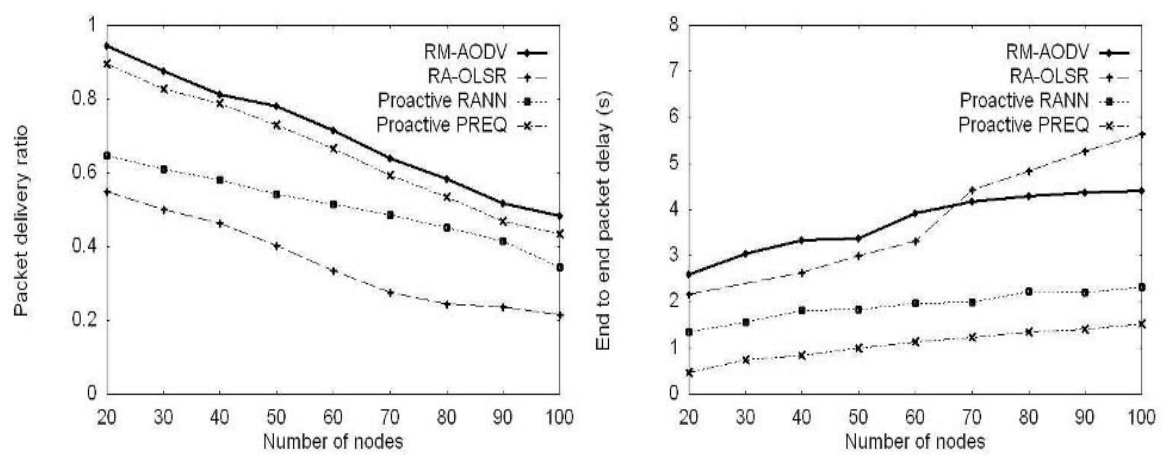

Fig. 10 Packet delivery ratio as a function of Fig. 11 End to end packet delay as a function of number of nodes number of nodes
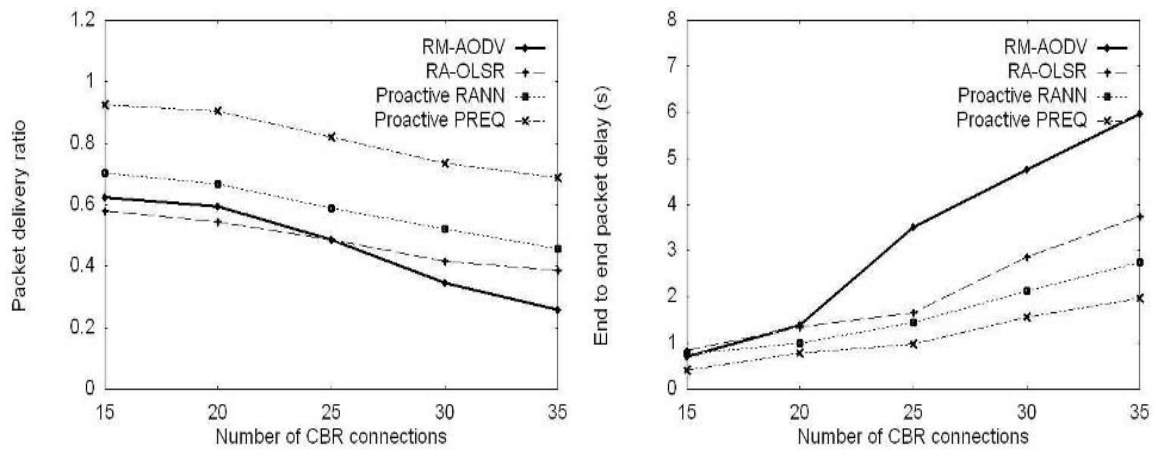

Fig. 12 Packet delivery ratio as a function of Fig. 13 End to end packet delay as a function of number of CBR connections number of CBR connections

proactive nature. Moreover, Proactive RANN has less delay then RA-OLSR for two reasons . First, Proactive RANN has less routing overhead. Second, in RA-OLSR, MPR are selected by an MP following some conditions. The first condition imposes that all strict two hops neighbors should be reached. The last condition requires that path metric (ALM) toward a strict two hops neighbors may be optimal. The conclusion from this is that, routes selected by RA-OLSR can not be optimal in term of end to end delay.

Figures 12 and 13 present the evaluation of path selection protocols by varying number of CBR connections intended to Root. We observe that the two proactive modes of HWMP have consistently better performance in term of packet delivery ratio and end to end packet delay. Indeed, Proactive PREQ and Proactive RANN are proactive path selection protocols, so routes are immediately available in routing table. In addition, they have less routing overhead then RA-OLSR and RM-AODV. 


\section{Conclusion}

This paper presented a detailed overview of the proposed path selection protocols of the upcoming IEEE 802.11s standard for WLAN mesh networks. In fact, the configurability of the default routing protocol HWMP and the extensibility framework for the routing with RA-OLSR as optional standardized routing protocol gives IEEE 802.11 s a broad applicability to many usage scenarios of wireless networks. We have investigated simulations to compare these path selection protocols. We have compared HWMP with its different modes to RA-OLSR. Our experiments and simulations have shown that the choice of a path selection protocol among IEEE 802.11s routing protocols is a difficult task, thus there is no protocol suitable for all cases. Indeed, each protocol improves good performance in certain conditions of network and traffic. For example, RM-AODV is more suitable in case where there are a small number of data connections. However, RA-OLSR is more appropriate in case where data traffic is distributed over the network and the number of nodes is not important. Finally, Proactive RANN and Proactive PREQ can provide good results in a network where most of the traffic is sent to Root. In conclusion, the two protocols complement each other, providing advantages in different domains. It is clear, that neither of the two protocol outperforms the other in all cases, and therefore, there is a need to keep both solutions available. For our future work, we plan to study the path selection protocols in a network using multi-channel technology to improve the network capacity. In addition, we project to study the effect of the ALM metric on the choice of the route and to see if it can take into account all the characteristics of mesh networks such as interference.

\section{References}

1. IEEE P802.11s/D1.08, Draft Amendment to Standard for Information Technology, January 2008.

2. Akyildiz, Wang. X and Wang. W: Wireless mesh networks: a survey, Computer Networks Elsevier Science, January. 2005.

3. Aoki, Takeda, Yagyu, and Yamada: IEEE 802.11s Wireless LAN Mesh Network Technology, NTT DoCoMo Tech J journal, 2006.

4. Bahr: Proposed routing for IEEE 802.11s WLAN mesh networks.(2006). In Proceedings of ACM International Conference, 2006.

5. Perkins, Belding-Royer and Das: Ad hoc on-demand distance vector (AODV) routing.(2003). In: IETF RFC 3561, July 2003.

6. Clausen, Jacquet and Laouiti: Optimized Link State Routing Protocol.(2003). In: IETF Internet RFC 3626, 2003.

7. Pei, Gerla, and Chen: Fisheye State Routing in Mobile Ad Hoc Networks.(2000). In InternetDraft, November 2000.

8. Zeng, Bagrodia and Gerla: GloMoSim: a Library for the Parallel Simulation of Large-scale Wireless Networks (1998). In Proceedings of PADS, 1998. 